\title{
Short communication: Expression and alternative splicing of POU1F1 pathway genes in preimplantation bovine embryos
}

\author{
J. Laporta, ${ }^{*}$ A. Driver, ${ }^{*}$ and H. Khatib*1 \\ *Department of Dairy Science, University of Wisconsin-Madison, Madison 53706 \\ †Department of Animal Production and Pastures, Universidad de la República (UDELAR), 12300 Montevideo, Uruguay
}

\begin{abstract}
Early embryo loss is a major contributing factor to cow infertility and that 70 to $80 \%$ of this loss occurs between d 8 and 16 postfertilization. However, little is known about the molecular mechanisms and the nature of genes involved in normal and abnormal embryonic development. Moreover, information is limited on the contributions of the genomes of dams and of embryos to the development and survival of preimplantation embryos. We hypothesized that proper gene expression level in the developing embryo is essential for embryo survival and pregnancy success. As such, the characterization of expression profiles in early embryos could lead to a better understanding of the mechanisms involved in normal and abnormal embryo development. To test this hypothesis, 2 d-8 embryo populations (degenerate embryos and blastocysts) that differed in morphology and developmental status were investigated. Expression levels of POU1F1 pathway genes were estimated in 4 sets of biological replicate pools of degenerate embryos and blastocysts. The OPN and STAT5A genes were found to be upregulated in degenerate embryos compared with blastocysts, whereas STAT5B showed similar expression levels in both embryo groups. Analysis of splice variants of $O P N$ and STAT5A revealed expression patterns different from the total expression values of these genes. As such, measuring expression of individual transcripts should be considered in gene expression studies.
\end{abstract}

Key words: embryo development, gene expression, alternative splicing, fertility

\section{Short Communication}

Infertility is a worldwide problem and a major cause of economic loss in the global dairy herd. Strong evidence exists of an association between selection for production traits and low pregnancy rates of high-

Received January 2, 2011.

Accepted April 21, 2011.

${ }^{1}$ Corresponding author: hkhatib@wisc.edu producing dairy cows (Dobson et al., 2007). Therefore, reproductive performance has recently been included in the selection indices of breeding organizations (Miglior et al., 2005). Low fertilization rate and embryonic mortality are considered the most significant factors contributing to failed pregnancies in dairy cows (Santos et al., 2004; Morris and Diskin, 2008). Although genetic factors account for an appreciable portion of the decline in fertility (Shook, 2006; Weigel, 2006; Veerkamp and Beerda, 2007), the identification of such factors has been challenging. To cope with this challenge, an in vitro fertilization system was established in which fertilization rate and embryonic survival - the 2 primary components of pregnancy - could be measured accurately and associated with genes affecting these traits. Indeed, the effectiveness of this in vitro fertilization system in identifying significant associations of genes and pathways with fertilization and embryonic survival rates has been demonstrated in previous studies (Khatib et al., 2008a,b, 2009a,b; Driver et al., 2009; Wang et al., 2009; Huang et al., 2010). Collectively, these studies focused on the contribution of the dam's genotype to the success of the fertilization process and embryonic development. Using the candidate pathway approach and dams' genotypic data, single genes in the pituitary-specific positive transcription factor 1 (POU1F1) pathway and their interactions revealed a significant contribution to the variation in fertilization and embryonic survival rates (Khatib et al., 2009a).

The questions of how much the dam contributes to early embryonic development and how much the embryo itself contributes remain to be answered. As such, the objective of this study was to evaluate the expression profiles of the POU1F1 pathway genes (Khatib et al., 2009a) in preimplantation embryos that differ in morphology and developmental status using quantitative real-time PCR (qRT-PCR). The STAT5B gene was included because of its high structural and functional similarity to STAT5A (Seyfert et al., 2000). Day 8 embryos were chosen for this study because this developmental stage signals the start of differentiation of the embryonic cells into trophectoderm and inner cell mass and the formation of blastocyst (Morris and 
Table 1. Primers used for total expression and alternative splicing analysis using quantitative real-time-PCR

\begin{tabular}{|c|c|c|c|}
\hline Sequence & Forward primer $\left(5^{\prime}-3^{\prime}\right)$ & Reverse primer $\left(5^{\prime}-3^{\prime}\right)$ & $\begin{array}{l}\text { Product } \\
\text { size (bp) }\end{array}$ \\
\hline \multicolumn{4}{|l|}{ Gene } \\
\hline$G A D P H$ & TGCCCAGAATATCATCCC & AGGTCAGATCCACAACAG & 134 \\
\hline GHR & ATGGCGGTATTGTGGATCAT & CTGTATTGGGTGGTGGTTCC & 150 \\
\hline$O P N$ & TCCTAACACCACAGAATT & GTTATCATCATCGTCATCTA & 122 \\
\hline POU1F1 & TTCTGCAACTCTGCCTCTGA & CCATAGGTCGATGACTGGT & 148 \\
\hline STAT5A & CCCAGCCTCACTATAACATG & GCTCCAGACTGTCCATTG & 138 \\
\hline STAT5B & GTT CCA TGC GAG CCT GCC AC & GTG CCA CCT ACA TGG ACC AG & 125 \\
\hline$U T M P$ & CATCCTGTGTCTTGTTAA & GTTCTGTCTTTCTCATCA & 137 \\
\hline \multicolumn{4}{|l|}{ Transcript $^{1}$} \\
\hline STAT5A-794 & GAC TCC ACC CAC GAG CAG & GGG ACA GGA CAC CAC GAC & 210 \\
\hline STAT5A-680 & AAGATCAAGCTGGGGCACT & CAACCAGGATCCCAGCAG & 154 \\
\hline OPN-201 & GACTGAAGTCAAGATCTAAG & GAA TCA CAG CAC AAA CAG & 82 \\
\hline
\end{tabular}

${ }^{1}$ Signal transducer activator transcript 5A (STAT5A) transcripts 794 and 690 and osteopontin (OPN) transcripts 201 and 278.

Diskin, 2008). Fertilization and embryo culture were as described in Khatib et al. (2008b). Semen samples from 2 bulls were used for fertilization. Before addition to oocytes, sperm underwent Percoll separation (45-90\% gradient) and were adjusted to a final concentration of $1 \times 10^{6} / \mathrm{mL}$ (Parrish et al., 1995). By d 5 of development, bovine embryos should attain approximately 16 to 32 cells and show signs of cellular compaction, deeming them as morulas. Embryos failing to show these characteristics were assumed to have been arrested in development and were excluded from analysis. Morulas were then cultured until d 8. Embryos that showed an evidence of a fluid-filled cavity (blastocoele), which gives rise to differentiation of the inner cell mass and trophectoderm, were referred to as blastocysts. In contrast, embryos that attained a compacted morula status by d 5 but failed the transition to blastocyst stage by d 8 were referred to as degenerate embryos. Embryos from each morphological group were collected in 2 pools of 20 and preserved in RNALater (Ambion, Austin, TX). Two sets of biological replicate pools from each sire were used. Thus, 4 degenerate and 4 blastocyst embryo pools were used in the expression analysis.

Primers (Table 1) were designed to amplify fragments spanning more than one exon to exclude the possibility of genomic DNA contamination in the qRT-PCR reactions using the Beacon Designer software (Premier Biosoft International, Palo Alto, CA). All samples were run in quadruplicate. Analysis of gene expression levels was conducted using the $2^{-\Delta \Delta \mathrm{Ct}}$ method (Livak and Schmittgen, 2001). For differential expression analysis between the embryo groups, normalized gene expression values $(\Delta \mathrm{Ct})$ were analyzed using a general linear model that included the fixed effects of the bull (2 levels), the type of embryo (blastocyst or degenerate), and the random effect of pool (8 levels). Association between the normalized gene expression and the type of embryo was tested using a likelihood ratio test by comparing this model to a reduced model without the embryo effect. The mean and range of the fold change for each gene were calculated as $2^{-\Delta \Delta C t}$ using the estimated $\Delta \Delta \mathrm{Ct}$ value \pm standard error.

The qRT-PCR analysis revealed lack of expression of $G H, G H R, P R L$, and PRLR in embryos. Expression levels of POU1F1 and UTMP were very low, and accurate measures of these genes could not be obtained. Figure 1 shows the expression differences (fold change) in embryos for STAT5A, STAT5B, and OPN. Both $S T A T 5 A$ and $O P N$ showed higher expression in degenerate embryos compared with blastocysts, 2.57- and 2.45-fold, respectively, whereas STAT5B expression levels were similar in the 2 embryo groups. In a previous study, the association of $O P N$ with fertilization rate and of $S T A T 5 A$ with fertilization and embryonic survival rates was reported using genotypic data of the dams (Khatib et al., 2009a). The upregulation of $O P N$ and STAT5A genes in degenerate embryos further validates these association studies and indicates that preimplantation embryos can be sensitive to gene dosage expression. In fact, several studies have shown that relatively small changes in gene expression can lead to growth abnormalities and cell death (Guillemot et al., 1995; Nollen and Morimoto, 2002).

Genotypes or genotypic interactions of $G H, G H R$, PRL, PRLR, POU1F1, and UTMP were found to be associated with fertility traits in the dams (Khatib et al., 2009a) but were lowly or not expressed in preimplantation embryos in this study. Most likely, these genes affect fertilization and embryo development through the maternal genome. However, for OPN and STAT5A, 


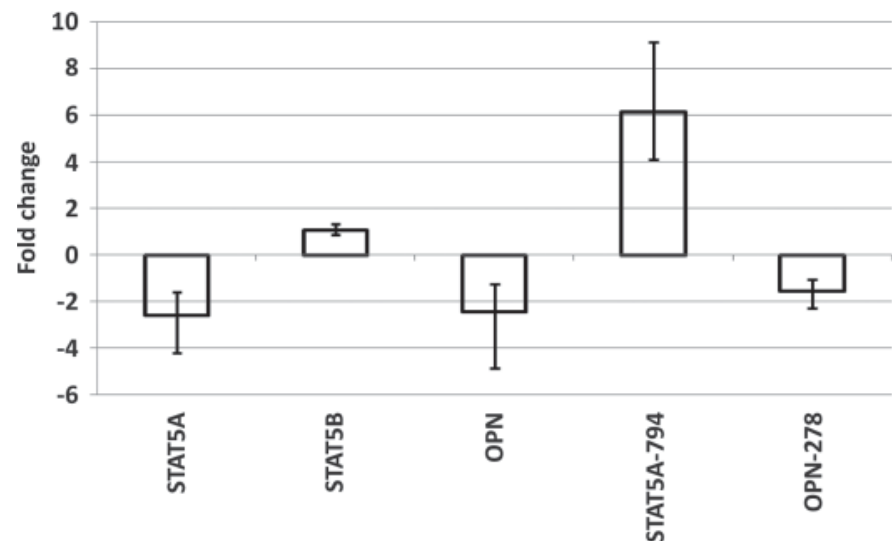

Figure 1. Fold change in expression of signal transducer activator transcript 5 (STAT5A and STAT5B), osteopontin (OPN), STAT5A transcript 794 (STAT5A-794), and OPN transcript 278 (OPN-278) in bovine embryos using quantitative real-time-PCR (qRT-PCR). Data are shown as mean \pm maximum and minimum fold changes Upregulation in degenerate or blastocyst embryos is represented by bars above or below the x-axis, respectively. The qRT-PCR was performed in 4 sets of biological replicates of blastocysts and degenerate embryo pools.

embryonic development is likely to be affected by both maternal genotype and the expression profiles of the embryos themselves. Indeed, 2 previous studies provide strong support for this assumption. In the first study, the occurrence of STAT5A alleles of maternal origin was found to be higher in blastocysts than in degenerate embryos (Khatib et al., 2009b). In a different study, significant associations of $S T A T 5 A$ genotypes with male fertility in a population of Holstein bulls was reported, testifying to the contribution of the male genome to embryo viability (Khatib et al., 2010).

The present study also tested if these differentially expressed bovine genes undergo alternative splicing. Alternative splicing is a process by which a single pre-mRNA transcript can produce 2 or more different mRNA molecules; it is considered to be a key factor in the evolution of phenotypic complexity (Blencowe, 2006). Wang et al. (2008) showed that over $92 \%$ of human multi-exon genes encode multiple transcripts. Search of the current Ensembl annotation of the bovine genome (http://uswest.ensembl.org/) revealed 2 transcripts for STAT5A (encoding 794 and $680 \mathrm{AA}$ ) and 3 transcripts for $O P N$ (encoding 201, 278, and $276 \mathrm{AA}$ ). Transcript-specific primers (Table 1) were designed in the transcripts of $S T A T 5 A$ and in 2 transcripts of $O P N$ and used in the qRT-PCR. For $O P N$, primers specific to transcript 276 could not be designed due to its high sequence similarity with transcript 278 . Although $O P N$ transcript 201 was not detected in embryos, transcript 278 showed a slightly higher expression (mean of 1.56 ) in degenerate embryos compared with blastocysts (Fig- ure 1). Transcript 680 of $S T A T 5 A$ was not detected in embryos, but was shown to be expressed in fetal lung, liver, kidney, brain, and chorion (data not shown). Surprisingly, transcript 794 of STAT5A showed a mean of 6.13-fold difference in blastocysts compared with degenerate embryos $(P=0.003)$, whereas the total expression of $S T A T 5 A$ was higher in degenerate embryos (Figure 1). Most likely, the observed total expression is an estimation of expression of multiple transcripts. It is possible that more alternative transcripts of STAT5A exist but have not been discovered yet given that the highly conserved human and mouse counterparts have 7 transcripts each. In addition, it is worth noting that the current annotation of the bovine genome is not complete. For example, we recently reported the discovery of thousands of alternative splicing events in other genes in cattle embryos and found some of these transcripts to be differentially expressed between blastocysts and degenerate embryos (Huang and Khatib, 2010). As such, given that the majority of multi-exonic genes have 2 or more transcripts, measuring expression of individual splice variants should be considered in gene expression studies.

In summary, expression analysis of the POU1F1 pathway genes showed that $G H, G H R, P R L, P R L R$, $P O U 1 F 1$, and $U T M P$ were lowly or not expressed in preimplantation embryos. However, given that genotyping of dams revealed significant associations with fertilization and embryonic survival rates, we conclude that embryonic development was affected primarily by the mother's genotype rather than by the genotype of the embryo, at least for these genes. In contrast, for $O P N$ and STAT5A, both the dam and embryo genotypes influence survival of the developing embryo. Thus, characterization of a dam- or embryo-specific genotype associated with embryo survival should be considered in the development of genetic markers for prediction of reproductive performance in cattle.

\section{ACKNOWLEDGMENTS}

This study was supported by the Robert Draper Technology Innovation Funding, Graduate School, University of Wisconsin. Jimena Laporta was supported by the National Agency of Investigation and Innovation, Uruguay. The authors thank Francisco Peñagaricano (University of Wisconsin-Madison) for the statistical analysis.

\section{REFERENCES}

Blencowe, B. J. 2006. Alternative splicing: New insights from global analyses. Cell 126:37-47.

Dobson, H., R. Smith, M. Royal, Ch. Knight, and I. Sheldon. 2007. The high-producing dairy cow and its reproductive performance. Reprod. Domest. Anim. 42(Suppl. 2):17-23. 
Driver, A. M., W. Huang, S. Gajic, R. L. Monson, G. J. Rosa, and H. Khatib. 2009. Effects of the progesterone receptor variants on fertility traits in cattle. J. Dairy Sci. 92:4082-4085.

Guillemot, F., T. Caspary, S. M. Tilghman, N. G. Copeland, D. J. Gilbert, N. A. Jenkins, D. J. Anderson, A. L. Joyner, J. Rossant, and A. Nagy. 1995. Genomic imprinting of Mash2, a mouse gene required for trophoblast development. Nat. Genet. 9:235-242.

Huang, W., and H. Khatib. 2010. Comparison of transcriptomic landscapes of bovine embryos using RNA-Seq. BMC Genomics 11:711.

Huang, W., B. W. Kirkpatrick, G. J. Rosa, and H. Khatib. 2010. A genome-wide association study using selective DNA pooling identifies candidate markers for fertility in Holstein cattle. Anim. Genet. $41: 570-578$.

Khatib, H., W. Huang, X. Wang, A. H. Tran, A. B. Bindrim, V. Schutzkus, R. L. Monson, and B. S. Yandell. 2009a. Single gene and gene interaction effects on fertilization and embryonic survival rates in cattle. J. Dairy Sci. 92:2238-2247.

Khatib, H., C. Maltecca, R. L. Monson, V. Schutzkus, and J. J. Rutledge. 2009b. Monoallelic maternal expression of STAT5A affects embryonic survival in cattle. BMC Genet. 10:13.

Khatib, H., C. Maltecca, R. L. Monson, V. Schutzkus, X. Wang, and J. J. Rutledge. 2008a. The fibroblast growth factor 2 gene is associated with embryonic mortality in cattle. J. Anim. Sci. 86:2063-2067.

Khatib, H., R. L. Monson, W. Huang, R. Khatib, V. Schutzkus, H. Khateeb, and J. J. Parrish. 2010. Short communication: Validation of in vitro fertility genes in a Holstein bull population. J. Dairy Sci. 93:2249-2259.

Khatib, H., R. L. Monson, V. Schutzkus, D. M. Kohl, G. J. Rosa, and J. J. Rutledge. 2008b. Mutations in the STAT5A gene are associated with embryonic survival and milk composition in cattle. J. Dairy Sci. 91:784-793.

Livak, K. J., and T. D. Schmittgen. 2001. Analysis of relative gene expression data using real-time quantitative PCR and the 2(-delta delta $\mathrm{C}(\mathrm{T})$ ) method. Methods 25:402-408.

Miglior, F., B. L. Muir, and B. J. Van Doormaal. 2005. Selection indices in Holstein cattle of various countries. J. Dairy Sci. 88:12551263 .
Morris, D., and M. Diskin. 2008. Effect of progesterone on embryo survival. Animal 2:1112-1119.

Nollen, E. A., and R. I. Morimoto. 2002. Chaperoning signaling pathways: Molecular chaperones as stress-sensing 'heat shock' proteins. J. Cell Sci. 115:2809-2816.

Parrish, J. J., A. Krogenaes, and J. L. Susko-Parrish. 1995. Effect of bovine sperm separation by either swim-up or Percoll method on success of in vitro fertilization and early embryonic development. Theriogenology 44:859-869.

Santos, J. E. P., W. W. Thatcher, R. C. Chebel, R. L. A. Cerri, and K. N. Galvao. 2004. The effect of embryonic death rates in cattle on the efficacy of estrus synchronization programs. Anim. Reprod. Sci. 82-83:513-535.

Seyfert, H. M., C. Pitra, L. Meyer, R. M. Brunner, T. T. Wheeler, A. Molenaar, J. Y. McCracken, J. Herrmann, H. J. Thiesen, and M. Schwerin. 2000. Molecular characterization of STAT5A- and STAT5B-encoding genes reveals extended intragenic sequence homogeneity in cattle and mouse and different degrees of divergent evolution of various domains. J. Mol. Evol. 50:550-561.

Shook, G. E. 2006. Major advances in determining appropriate selection goals. J. Dairy Sci. 89:1349-1361.

Veerkamp, R. F., and B. Beerda. 2007. Genetics and genomics to improve fertility in high producing dairy cows. Theriogenology 68:S266-S273.

Wang, E. T., R. Sandberg, S. Luo, I. Khrebtukova, L. Zhang, C. Mayr, S. F. Kingsmore, G. P. Schroth, and C. B. Burge. 2008. Alternative isoform regulation in human tissue transcriptomes. Nature 456:470-476.

Wang, X., V. Schutzkus, W. Huang, G. J. Rosa, and H. Khatib. 2009. Analysis of segregation distortion and association of the bovine FGF2 with fertilization rate and early embryonic survival. Anim. Genet. 40:722-728.

Weigel, K. A. 2006. Prospects for improving reproductive performance through genetic selection. Anim. Reprod. Sci. 96:323-330. 